\title{
CONOCIMIENTOS Y ACTITUDES SOBRE LA TRANS- MISIÓN DE LA TUBERCULOSIS EN ESTUDIANTES DE ODONTOLOGÍA Y TERAPIA RESPIRATORIA
}

Sonia Elena Pineda ${ }^{1}$, Adriana Posada López ${ }^{2}$, Blanca Ros Mary Mendoza Pinzón ${ }^{3}$, Laura Giraldo Quintero 4

\section{Resumen}

Introducción. La tuberculosis sigue siendo un problema importante de salud pública, que afecta tanto a trabajadores como estudiantes de la salud, quienes deben tener unos conocimientos adecuados y sólidos para asegurar una adecuada prevención y control de la enfermedad. Objetivo. Evaluar los conocimientos y actitudes sobre la transmisión de la tuberculosis en estudiantes de odontología y terapia respiratoria. Métodos. Estudio descriptivo realizado con 291 estudiantes de los programas de odontología y terapia respiratoria de una institución de educación superior de la ciudad de Medellín, quienes se encontraban en práctica clínica al momento del estudio. Ellos respondieron una encuesta que contenía información sociodemográfica, conocimientos y actitudes frente a la transmisión de la tuberculosis. Se realizó un análisis descriptivo y bivariado. Resultados. El 57,7\% de los estudiantes de odontología y el $42,3 \%$ de los estudiantes de terapia respiratoria consideran que la tuberculosis es trasmitida por una bacteria, y respecto a si la tos es considerada un mecanismo de transmisión, el 89,2\% de los estudiantes de odontología dijo que sí y el 10,8\% de los estudiantes de terapia respondieron que no. Conclusiones. La mayoría de los estudiantes respondieron que el factor etiológico de la tuberculosis es una bacteria y en en cuanto a la vacunación, la mayoría considera que es importante para la protección.

\section{Palabras clave: conocimiento, Mycobacterium tuberculosis, actitud, (DeCS).}

1. Magíster en Salud Pública. Especialista en Ciencias Forenses. Bacterióloga y Laboratorista Clínica. Docente Facultad de Odontología. Fundación Universitaria Autónoma de las Américas, Sede Medellín.sonia.pineda@uam.edu.co

2. Magíster en Epidemiología. Odontóloga. Docente Facultad de Odontología, Fundación Universitaria Autónoma de las Américas. Sede Medellín. adriana.posada@uam.edu.co

3. Terapeuta Respiratoria. Facultad de Terapia Respiratoria. Fundación Universitaria Autónoma de las Américas. Sede Medellín. blanca.mendoza@uam.edu.co

4. Estudiante de Odontología. Facultad de Odontología. Fundación Universitaria

Autónoma de las Américas. Sede Medellín. laura.giraldoq@uam.edu.co 


\section{KNOWLEDGE AND ATTITUDES ON THE TRANSMISSION OF TUBERCULOSIS IN DENTISTRY AND RESPIRATORY THERAPY STUDENTS}

Sonia Elena Pineda, Adriana Posada López, Blanca Ros Mary Mendoza Pinzón, Laura Giraldo Quintero

\section{Abstract}

Introduction. Tuberculosis continues to be a major public health issue, affecting both workers and students of health, who must have adequate and solid knowledge to ensure adequate prevention and control of the disease. Objective. To evaluate the knowledge and attitudes on the transmission of tuberculosis in students of dentistry and respiratory therapy. Methods. A descriptive study was carried out with 291 students from the dentistry and respiratory therapy programs of a higher education institution in the city of Medellín, who were in clinical practice at the time of the study. They answered a survey based on sociodemographic information, knowledge and attitudes regarding the transmission of tuberculosis. A descriptive and bivariate analysis was carried out. Results. $57.7 \%$ of dentistry students and $42.3 \%$ of respiratory therapy students consider that tuberculosis is transmitted by a bacterium, and if cough is considered a transmission mechanism, $89.2 \%$ of dentistry students said that it was and $10.8 \%$ of the therapy students answered no. Conclusions. The majority of the students answered that the etiological factor of tuberculosis is a bacterium and regarding to vaccination, most of them consider it is important for protection. 


\section{CONHECIMENTO E ATITUDES NA TRANSMISSÃO DA TUBERCULOSE EM ESTUDANTES DE ODONTOLOGIA E TERAPIA RESPIRATÓRIA}

Sonia Elena Pineda, Adriana Posada López, Blanca Ros Mary Mendoza Pinzón, Laura Giraldo Quintero

\section{Resumo}

Introdução. A tuberculose continua sendo um grande problema de saúde pública, afetando tanto os trabalhadores quanto os estudantes de saúde, que devem ter conhecimentos adequados e sólidos para garantir a prevenção e o controle adequados da doença. Objetivo. Avaliar os conhecimentos e atitudes sobre a transmissão da tuberculose em estudantes de odontologia e fisioterapia respiratória. Métodos. Foi realizado um estudo descritivo com 291 estudantes dos cursos de odontologia e fisioterapia respiratória de uma instituição de ensino superior da cidade de Medellín, que estavam em prática clínica na época do estudo. Eles responderam a uma pesquisa que continha informações sociodemográficas, conhecimentos e atitudes em relação à transmissão da tuberculose. Uma análise descritiva e bivariada foi realizada. Resultados. 57,7\% dos estudantes de odontologia e $42,3 \%$ dos estudantes de fisioterapia respiratória consideram que a tuberculose é transmitida por bactérias; com respeito a se a tosse é considerada um mecanismo de transmissão, $892 \%$ dos estudantes de odontologia disseram que sim e $10,8 \%$ dos estudantes de terapia responderam que não. Conclusões. A maioria dos estudantes respondeu que o fator etiológico da tuberculose é uma bactéria e a maioria considera a vacinação importante para a proteção. 


\section{Introducción}

La tuberculosis (TB) es la enfermedad infectocontagiosa crónica y multisistémica más frecuente en el mundo (1), causada por el Mycobacterium tuberculosis. Es una enfermedad prevenible y curable, sin embargo, en el mundo sigue siendo un problema de salud pública a nivel mundial, se calcula que una cuarta parte de la población mundial tiene tuberculosis latente (asintomática y no infectante) y 1 de cada 10 individuos expuestos adquieren la enfermedad (2). La TB es la novena causa de muerte y la primera por enfermedades infecciosas, por encima del VIH.

En 2016, la cifra estimada de muertes por TB fue de 1,3 millones y la de personas que contrajeron la TB fue de 10,4 millones: $90 \%$ adultos, el $65 \%$ del sexo masculino y en ese mismo año, 6,3 millones de nuevos casos de TB fueron notificados a nivel mundial (3). Con respecto a la TB multirresistente (MDR TB) definida como cepa de M. tuberculosis resistente in vitro a isoniazida y rifampicina, simultáneamente o combinado con otros fármacos antituberculosos,(4) representa actualmente un serio problema para el control mundial de la TB, por lo que el diagnóstico temprano de una TB activa y la detección precoz de cepas multi-resistentes es esencial para interrumpir su transmisión (5).

Teniendo en cuenta los objetivos de desarrollo del milenio (ONU, 2000), nuestro país, con el Plan Decenal de Salud Pública PDSP 2012 - 2021 presenta grandes retos que involucran tanto al sector salud como a otros sectores, para crear condiciones que garanticen el bienestar integral y la calidad de vida en Colombia; siendo la tuberculosis considerada una de las prioridades a intervenir en el marco de la dimensión vida saludable y enfermedades transmisibles en el componente de enfermedades emergentes, re-emergentes y que dentro de sus objetivos se encuentra el vigilar, controlar, y contener la resistencia a los antimicrobianos implicando plantear estrategias de intervención colectiva e individual (6).

El riesgo biológico por exposición laboral a infecciones causadas por diversos agentes, especialmente virus y bacterias, se ha convertido en una amenaza para el personal de salud, mientras realizan sus actividades; en especial, si se considera el número creciente de infecciones emergentes y re-emergentes (influenza AH1N1 y la tuberculosis), el VIH/SIDA $\mathrm{y}$ otras infecciones transmitidas por vía sanguínea (hepatitis B y C) (7).

En el Informe Quinquenal Epidemiológico Nacional (IQEN) del año 2013, llamado Tuberculosis en personal de salud Colombia 2008 -2012, se estimó que en Colombia, en 2012 se registraron 7298 casos nuevos de TB en trabajadores de la salud con baciloscopia positiva. Antioquia y Valle del Cauca con 35.8\% fueron los departamentos con el mayor número de casos notificados con TB (8). La vía de transmisión más frecuente es la 'vía aérea', a través de la inhalación de goticulas (partículas menores a 5 micras) procedentes de las secreciones respiratorias emitidas al toser, estornudar $y$ hablar, o bien por la inhalación de partículas de polvo que contengan el agente infeccioso (2). 
Los trabajadores de la salud son un recurso fundamental en la atención de los pacientes con TB, tanto en su orientación diagnóstica y terapéutica como en las acciones de promoción y prevención (9). El grupo de trabajadores de la salud es vulnerable a adquirir la enfermedad y su riesgo ocupacional está determinado directamente por la exposición a pacientes infectados, por lo que es de declaración obligatoria a nivel nacional (EDO), porque además es una enfermedad ocupacional que se presenta en aquellas personas que la contraen a causa de su trabajo; la TB pulmonar, la de vías respiratorias superiores (laringe y cavidad oral) y la TB extrapulmonar con abscesos o lesión abierta con alta concentración de bacilos o drenaje extenso, son las formas más importantes implicadas en la transmisión de TB a trabajadores sanitarios (10).

En áreas clínicas como urgencias, consulta externa, neumología, salas de terapia respiratoria, laboratorios de microbiología e histopatología, las posibilidades de infección son más altas, porque el aislamiento y el tratamiento no se han implementado, debido a que los pacientes aún no han sido diagnosticados. Varios estudios han demostrado que los trabajadores del área de la salud tienen un riesgo 10 a 100 veces mayor, en relación con la población general, lo mismo sucede en los internados y las prácticas clínicas de los estudiantes del área de la salud, ya que son entornos de alto riesgo para la infección de TB, pues su exposición es directa y frecuente, lo que hace esto más factible, sobre todo en los países en vía de desarrollo (11).

Frente a los conocimientos del personal de salud, incluyendo estudiantes y pro- fesionales, la literatura refiere que deben ser idóneos, adecuados y sólidos para asegurar el éxito de la prevención, el diagnóstico, la vigilancia y el control de la TB. Estos deben garantizar una adecuada capacitación y orientación a las personas con la enfermedad, a sus familias y las comunidades, para un adecuado y seguro tratamiento anti-tuberculoso (12). Además de las buenas prácticas de trabajo, la educación de los pacientes y coordinación entre dichos programas $(13,14)$.

En muchos lugares del mundo, la práctica clínica dentro del plan de estudios de medicina y de ciencias de la salud se realiza en los últimos años y corresponde a la etapa en la cual los alumnos integran y consolidan los conocimientos que adquirieron durante los años previos. Adicionalmente, ellos mantienen un contacto más estrecho con los pacientes, aumentando el riesgo de adquirir tuberculosis nosocomial(9). El mayor índice se presenta en el personal de enfermería, terapia respiratoria, residentes y estudiantes de pregrado de medicina.

De igual manera, los estudiantes de odontología, quienes se preparan para prestar atención en salud, están expuestos a una variedad de microorganismos, debido al contacto directo o indirecto con el instrumental, el equipo, aerosoles y superficies contaminadas, así como también a los fluidos corporales de los pacientes, por lo que se hace necesario tomar diferentes medidas de protección para prevenir la infección cruzada(15).

Los estudiantes se encuentran expuestos al agente infeccioso, cuando brindan 
por la cual se hace necesario un mayor conocimiento acerca de la enfermedad, las medidas de protección y las políticas laborales adecuadas en los establecimientos de salud. El trabajador del área de salud debe conocer los síntomas de la tuberculosis y someterse a un examen médico que incluya pruebas para detectarla, por lo menos, una vez al año (16). La aplicación de las precauciones universales como es el conocimiento de la enfermedad y las medidas de bioseguridad constituye un aspecto vital en la práctica cotidiana (17).

Actitudes y conocimientos escasos e inadecuados de los estudiantes con respecto a la transmisión de la tuberculosis podría contribuir a generar conceptos errados que pueden poner en riesgo la integridad de los estudiantes y de los pacientes.

Por lo anteriormente expuesto, el objetivo de esta investigación fue identificar los conocimientos y actitudes sobre la transmisión de la tuberculosis en estudiantes de odontología y terapia respiratoria.

\section{Metodología}

\section{Diseño}

Se realizó un estudio de tipo descriptivo cuantitativo, donde se aplicó un instrumento que contenía información acerca de las características sociodemográficas de los estudiantes, además de preguntas relacionadas con los conocimientos y actitudes sobre la transmisión de la tuberculosis.
La población estuvo conformada por 291 estudiantes de los programas de odontología y terapia respiratoria de una institución de educación superior de la ciudad de Medellín. Se realizó un muestreo no probabilístico. Los criterios de inclusión consideraron a estudiantes matriculados en los cursos de Atención Clínica Integral para el caso de los estudiantes de odontología y de cuarto a noveno semestre del programa de Terapia Respiratoria matriculados en los cursos de práctica clínica, ambos grupos mayores de 18 años y que libremente decidieron participar de la investigación. Como criterio de exclusión se definió el no realizar trabajo clínico y no desear participar de la investigación. Para la obtención de la información, el equipo de trabajo se dirigió a cada uno de los grupos y se les dio a conocer los objetivos de la investigación, se firmó el consentimiento informado y se procedió con el desarrollo de la respectiva encuesta.

\section{Instrumento}

El instrumento para la recolección de la información se estructuró de la siguiente forma:

1. Características de los estudiantes: Edad, sexo, programa académico.

2. Identificación de los conocimientos: Etiología de la TB, mecanismo de transmisión, ¿Cómo se hace la búsqueda activa?, formas infectantes y el diagnóstico.

3. Identificación de las actitudes: ¿Cómo se siente cuando se atiende a una apersona con TB? ¿Considera importante la educación de la familia del paciente con TB? 
Posteriormente, se realizó una prueba piloto para identificar si existía la claridad en la formulación de las preguntas y el tiempo estimado para responder la encuesta.

Una vez recolectada la información se digitó en SPSS versión 21, dicha información fue revisada por los investigadores para minimizar los errores durante el proceso de la digitación. Se realizó un análisis descriptivo con cada una de las variables de interés con sus respectivas frecuencias para las cualitativas, y para las variables cuantitativas se calcularon media y desviación estándar (DE). Además, se buscó posibles relaciones entre las variables de interés, mediante la aplicación de las pruebas de Chi- cuadrado. Esta investigación se enmarcó bajo los principios de la declaración de Helsinki y la Resolución 8430 de 1993 del Ministerio de salud de Colombia, la cual la clasifica como una investigación sin riesgo. A los participantes se les explicaron los objetivos de la investigación y la autonomía que tenían, si en algún momento no deseaban continuar con la investigación, y esto no les afectaría en nada el vínculo con la institución. Además, el proceso contó con criterios de confidencialidad $\mathrm{El}$ proyecto fue aprobado por el comité de ética institucional, mediante el Acta del 9 de octubre de 2016. Los estudiantes firmaron el consentimiento informado.

\section{Resultados}

\section{Características sociodemográficas}

La población estuvo conformada por 291 estudiantes, quienes tenían una edad promedio de 22,7 años ( $\mathrm{DE}=3,6$ años). El 63,2\%(184) correspondían al Programa de Odontología y el 36,8\%(107) al Programa de Terapia Respiratoria. La frecuencia por sexo indicó que el 84,9\% (247) eran mujeres. Con relación al estado civil se encontró que el $89,3 \%(260)$ eran solteros.

Tabla 1. Características sociodemográficas de los estudiantes.

\begin{tabular}{l|l|l}
\hline Variables & $\mathbf{n}$ & $\%$ \\
\hline Edad & 172 & 59,1 \\
$18-22$ & 95 & 32,6 \\
$23-27$ & 24 & 8,2 \\
28 o más & & \\
Sexo & & \\
Hombre & 44 & 15,1 \\
Mujer & 247 & 84,9 \\
Estado civil & &
\end{tabular}




\begin{tabular}{lcc} 
Solteros & 260 & 89,3 \\
Casados & 21 & 72,2 \\
Unión libre & 10 & 3,4 \\
Programa Académico & & \\
Odontología & 184 & 63,2 \\
Terapia Respiratoria & 107 & 36,8 \\
\hline
\end{tabular}

Fuente: Datos de la investigación.

\section{Conocimientos sobre la transmisión de la tuberculosis}

Con relación a los conocimientos que manifestaron los estudiantes sobre la TB se encontró que el 72,0\% de los estudiantes de odontología y el 89,7\% de los estudiantes de terapia respiratoria consideran que la TB es trasmitida por una bacteria, al indagar si la tos de una persona infectada con TB es considerado un mecanismo de transmisión de la enfermedad, el $82,1 \%(151)$ de los estudiantes de odontología y el 96,3\%(103)de los estudiantes de terapia respondieron que sí.

Respecto a si se debe realizar búsqueda activa de tuberculosis en pacientes con
VIH, el 63,6\% (117) de los estudiantes de odontología y el 25,2\%(27) de los estudiantes de terapia reportaron que no era necesario.

Con relación a la pregunta acerca de si convivir con una persona que padezca $\mathrm{TB}$, transmite dicha enfermedad, el $57,6 \%$ de los estudiantes de odontología y el 79,4\% de los de terapia consideran que sí. En cuanto a la vacunación, el $77,6 \%$ de los estudiantes de odontología y el $64,5 \%$ de terapia respiratoria, refirieron que esta protege contra la TB pulmonar. Los anteriores resultados mostraron diferencias estadísticamente significativas $(\mathrm{p}<=0,005)$ (Tabla 2).

Tabla 2. Conocimientos sobre la transmisión de TB según el programa al que pertenecen los estudiantes.

\begin{tabular}{cc|c|c|c|c}
\hline \multicolumn{2}{c|}{$\begin{array}{c}\text { Odontología } \\
(\mathrm{n}=184)\end{array}$} & \multicolumn{2}{c|}{$\begin{array}{c}\text { Terapia } \\
\text { respiratoria } \\
(\mathrm{n}=107)\end{array}$} & $\begin{array}{c}\text { Valor } \\
\mathbf{p}\end{array}$ \\
\hline $\mathrm{n}$ & $\%$ & $\mathrm{n}$ & $\%$ & \\
\hline
\end{tabular}

\section{La TB es trasmitida por}

$\begin{array}{ccccc}49 & 26,9 & 10 & 9,3 & 0,002 \\ 2 & 1,1 & 1 & 1,0 & \end{array}$




\begin{tabular}{lc|c|c|c|c}
\hline & \multicolumn{2}{c|}{$\begin{array}{c}\text { Odontología } \\
(\mathrm{n}=184)\end{array}$} & \multicolumn{2}{|c|}{$\begin{array}{c}\text { Terapia } \\
\text { respiratoria } \\
(\mathrm{n}=107)\end{array}$} & $\begin{array}{c}\text { Valor } \\
\mathrm{p}\end{array}$ \\
\cline { 2 - 6 } & $\mathrm{n}$ & $\%$ & $\mathrm{n}$ & $\%$ & \\
\hline
\end{tabular}

La tos de una persona infectada con TB produce TB

Sí

No

Contacto con fluidos corporales

No

Compartir implementos de cocina y aseo

Sí

No

Se debe realizar búsqueda activa de la tuberculosis en personas con VIH

Sí

No

Se debe realizar búsqueda activa en personas con infecciones respiratorias

Sí

No

Gravedad de la enfermedad

\begin{tabular}{lccccc} 
Muy grave & 104 & 56,6 & 62 & 57,9 & \\
Grave & 79 & 42,9 & 45 & 42,1 & 0,734 \\
Poco grave & 1 & 0,54 & 0 & 0,0 & \\
\hline
\end{tabular}

Ser vecino de una persona con TB

trasmite la TB

Sí

No

$\begin{array}{lllll}141 & 76,6 & 58 & 54,2 & \\ 43 & 23,4 & 49 & 45,8 & \end{array}$

$\begin{array}{lllll}146 & 79,3 & 94 & 87,9 & 0,044\end{array}$

$\begin{array}{llll}67 & 36,4 & 80 & 74,8\end{array}$

$\begin{array}{llll}117 & 63,6 & 27 & 25,2\end{array}$

0,000

$\begin{array}{lllll}80 & 43,5 & 23 & 21,5 & 0,000 \\ 104 & 56,5 & 84 & 78,5 & \end{array}$




\begin{tabular}{cc|c|c|c|c}
\hline & \multicolumn{2}{c|}{$\begin{array}{c}\text { Odontología } \\
(n=184)\end{array}$} & $\begin{array}{c}\text { Terapia } \\
\text { respiratoria } \\
(n=107)\end{array}$ & $\begin{array}{c}\text { Valor } \\
p\end{array}$ \\
\cline { 2 - 5 } & $\mathrm{n}$ & $\%$ & $\mathrm{n}$ & $\%$ & \\
\hline
\end{tabular}

\section{La vacuna protege contra TB)}

$\begin{array}{lccccc}\text { Miliar } & 8 & 4,6 & 15 & 14,0 & \\ \text { Meníngea } & 30 & 17,2 & 23 & 21,5 & \\ \text { Pulmonar } & 135 & 77,6 & 69 & 64,5 & 0,019 \\ \text { Otra } & 1 & 0,6 & 0 & 0,0 & \end{array}$

Todas las formas de TB son infectantes

\begin{tabular}{lccccc} 
Sí & 106 & 57,6 & 60 & 56,1 & 0,447 \\
No & 78 & 46,4 & 47 & 43,9 & \\
$\begin{array}{l}\text { Los niños con TB generalmente no son } \\
\text { contagiosos }\end{array}$ & & & & & \\
Sí & 115 & 62,5 & 65 & 60,7 & 0,431 \\
No & 69 & 37,5 & 42 & 39,3 & \\
Conoce la estrategia Colombia libre de & & & & & \\
TB & 18 & 9,8 & 16 & 15,0 & 0,129 \\
Sí & 166 & 90,2 & 91 & 85,0 & \\
No & & & & & \\
\hline
\end{tabular}

Fuente: Datos de la investigación.

Tabla 3. Actitudes de los estudiantes frente a la transmisión de la TB según programa académico.

\begin{tabular}{|c|c|c|c|c|c|c|}
\hline \multirow[t]{2}{*}{ Pregunta } & \multirow[t]{2}{*}{$\begin{array}{l}\text { Progra- } \\
\text { ma aca- } \\
\text { démico }\end{array}$} & $\begin{array}{l}\text { Total- } \\
\text { mente de } \\
\text { acuerdo }\end{array}$ & $\begin{array}{c}\text { De } \\
\text { acuerdo }\end{array}$ & $\begin{array}{l}\text { En des- } \\
\text { acuerdo }\end{array}$ & $\begin{array}{c}\text { Total- } \\
\text { mente } \\
\text { en des- } \\
\text { acuerdo }\end{array}$ & \multirow[t]{2}{*}{$\begin{array}{l}\text { Valor } \\
\text { p }\end{array}$} \\
\hline & & n (\%) & n (\%) & n (\%) & n (\%) & \\
\hline $\begin{array}{l}\text { *Es interesante informar } \\
\text { y educar al paciente y su } \\
\text { familia acerca de la TB }\end{array}$ & $\begin{array}{c}\text { OD } \\
(n=183)\end{array}$ & $\begin{array}{l}163(89,1) \\
104(97,2)\end{array}$ & $\begin{array}{l}18(9,8) \\
2(1,9)\end{array}$ & $\begin{array}{l}2(1,1) \\
1(0.9)\end{array}$ & $\begin{array}{l}0(0,0) \\
0(0,0)\end{array}$ & 0,035 \\
\hline $\begin{array}{l}\text { Es incomodo atender a los } \\
\text { pacientes con TB }\end{array}$ & & $\begin{array}{c}47(25,6) \\
7(6,5)\end{array}$ & $\begin{array}{l}55(29,9) \\
28(26,2)\end{array}$ & $\begin{array}{l}65(35,3) \\
51(47,7)\end{array}$ & $\begin{array}{c}17(9,2) \\
21(19,6)\end{array}$ & 0,000 \\
\hline
\end{tabular}




\begin{tabular}{|c|c|c|c|c|c|c|}
\hline \multirow[t]{2}{*}{ Pregunta } & \multirow[t]{2}{*}{$\begin{array}{l}\text { Progra- } \\
\text { ma aca- } \\
\text { démico }\end{array}$} & $\begin{array}{l}\text { Total- } \\
\text { mente de } \\
\text { acuerdo }\end{array}$ & $\begin{array}{c}\text { De } \\
\text { acuerdo }\end{array}$ & $\begin{array}{l}\text { En des- } \\
\text { acuerdo }\end{array}$ & $\begin{array}{c}\text { Total- } \\
\text { mente } \\
\text { en des- } \\
\text { acuerdo }\end{array}$ & \multirow[t]{2}{*}{$\begin{array}{c}\text { Valor } \\
p\end{array}$} \\
\hline & & n (\%) & n (\%) & n (\%) & n (\%) & \\
\hline $\begin{array}{l}\text { Es importante indagar } \\
\text { sobre los efectos adversos } \\
\text { de los medicamentos en } \\
\text { pacientes con TB }\end{array}$ & & $\begin{array}{l}152(82,6) \\
85(79,4)\end{array}$ & $\begin{array}{l}30(16,4) \\
22 \\
(20,68)\end{array}$ & $\begin{array}{l}1(0,54) \\
0(0,0)\end{array}$ & $\begin{array}{l}1(0,54) \\
0(0,0)\end{array}$ & 0,587 \\
\hline $\begin{array}{l}\text { Para usted es un riesgo } \\
\text { entrevistar a un paciente } \\
\text { con TB }\end{array}$ & & $\begin{array}{l}37(20,1) \\
22(20,6)\end{array}$ & $\begin{array}{l}69(37,5) \\
47(43,9)\end{array}$ & $\begin{array}{l}56(30,4) \\
28(26,2)\end{array}$ & $\begin{array}{l}22(12,0) \\
10(9,3)\end{array}$ & 0,670 \\
\hline $\begin{array}{l}\text { Es necesario realizar el } \\
\text { examen de esputo a los } \\
\text { contactos del paciente } \\
\text { con TB }\end{array}$ & & $\begin{array}{l}119(64,7) \\
76(71,0)\end{array}$ & $\begin{array}{l}56(30,4) \\
26(24,3)\end{array}$ & $\begin{array}{l}7(3,8) \\
5(4,7)\end{array}$ & $\begin{array}{l}2(1,1) \\
0(0,0)\end{array}$ & 0,458 \\
\hline
\end{tabular}

Fuente: Datos de la investigación.

*número de personas que respondieron esta pregunta del programa de Odontología

Actitudes frente a la transmisión de la TB

En cuanto a las actitudes que tienen los estudiantes sobre la transmisión de la TB, según el programa que estudian, se encontró que el 97,2\% de TR está totalmente de acuerdo con que es interesante informar y educar al paciente y su familia acerca de la TB. El 25,6\% de los de OD dice que está totalmente de acuerdo con que es incómodo atender a los pacientes con TB, mientras que el 19,6\% de los estudiantes de TR dice que están totalmente en desacuerdo con esa afirmación. ( $p=0,0035$ y 0,000 respectivamente).

\section{Discusión de resultados}

La importancia del conocimiento en transmisión de la TB en el personal de salud en formación es una necesidad apremiante y se convierte en una forta- leza en la lucha contra la tuberculosis, en los diferentes países. La participación de los estudiantes de pregrado en el control de esta enfermedad tanto en la detección, como en el seguimiento al tratamiento, es parte fundamental para prevenir posibles contagios, ya que propicia comportamientos de autocuidado del personal sanitario, de los pacientes y las familias, lo que brinda soporte a los programas de control de la tuberculosis.

Para generar procesos académicos efectivos en el control de la tuberculosis, se debe garantizar la participación de los estudiantes en la asistencia clínica de pacientes enfermos y contar con el conocimiento de las estrategias nacionales de la lucha contra la tuberculosis, dando a conocer los programas e información acerca de las formas de contagio, tratamiento y prevención, por medio del reconocimiento de los sintomáticos respiratorios y la utilización de medidas de 
bioseguridad en el estudiante que está en práctica.

Para la prevención de la enfermedad, es necesario resaltar que existen políticas nacionales que son desconocidas para la mayor parte de los estudiantes, como se evidenció en esta investigación, donde se encontró que solo un $15 \%$ y $9.8 \%$ de los estudiantes de terapia respiratoria $y$ odontología, respectivamente conocía sobre el "Plan Estratégico Colombia Libre de Tuberculosis 2010-2015" para el logro de las metas propuestas en el marco de la estrategia global "Alto a la TB" (18).

Esta investigación concuerda con los hallazgos del estudio de Ortega y Col, 2015, en la cual se encontraron bajos aciertos en cuanto a los conocimientos de los estudiantes de enfermería acerca de la TB, en cuanto a etiología, utilidad de la vacuna y la búsqueda del sintomático respiratorio. En este estudio también se evidenciaron falencias en estos aspectos (17).

Al igual que en el trabajo de Abreu y Col, 2013(19), en el que se evidenció que la mayoría de los estudiantes de medicina desconocía las formas clínicas más frecuentes de la TB o sospechar de un individuo sintomático, los datos en el presente estudio arrojaron resultados similares en estos mismos aspectos y en un estudio de 'conocimientos y actitudes de las medidas de bioseguridad en estudiantes de odontología en Puno' se encontró que los estudiantes de odontología, al no tener un adecuado conocimiento sobre el riesgo de contagio de tuberculosis (15), no siempre toman las medidas de bioseguridad adecuadas.
Adicionalmente, los resultados también se relacionan con lo expresado en un estudio acerca de los conocimientos sobre tuberculosis en trabajadores de la salud, en una localidad de Bogotá, el cual (9), en respuestas erradas como "la tuberculosis es desconocida, viral, hereditaria" y considerar que, "a pesar de que se dé tratamiento a la enfermedad, sigue siendo transmisible". Estos resultados no se alejan de los observados durante la presente investigación, debido a que hubo fallas en la respuesta acerca de la transmisión de $\mathrm{TB}$, riesgo de contagio y también, en la generación de falsas creencias, mitos o temor que conducen a la perpetuación del estigma que llevan los pacientes con la enfermedad (9).

En el Informe de la Organización Panamericana de la Salud (20) se aclara que muchas de las brechas encontradas en la enseñanza de la tuberculosis en las facultades de salud, se solucionan revisando los planes de estudio y programas curriculares para garantizar que se imparta el tema de la TB, con base en la realidad y normas nacionales, ya que juegan un papel importante las universidades en el control de la tuberculosis, por medio del análisis de los contenidos curriculares existentes, la evaluación de su coherencia y de su compatibilidad con los objetivos de la política sanitaria y con el análisis, formulación, implantación y aplicación de la reglamentación profesional, con el fin de marcar diferencias entre lo que se enseña y se hace en la práctica para combatir la tuberculosis en Colombia. Esto se evidencia en el momento de aplicar los conocimientos sobre la enfermedad en la atención del paciente y en el control de la enfermedad, practicando las medidas de biose- 
guridad y la educación al paciente y su familia (20).

En un reciente estudio acerca de los "conocimientos, actitudes, prácticas y educación sobre tuberculosis, en estudiantes de una facultad en salud" realizado con un total de 193 estudiantes de diferentes áreas de la salud (15,5\% estudiantes de odontología), se encontró un conocimiento insuficiente sobre prevención de la TB, signos, síntomas, diagnóstico y tratamiento (11). Por su parte, los resultados en cuanto a los síntomas respiratorios y factores de riesgo de contagio fueron muy similares a los encontrados en la presente investigación .

\section{Conclusiones}

Los resultados de esta investigación reflejan falencias en los estudiantes que están en la práctica clínica respecto a conceptos etiopatogénicos y clínicos de la TB, al igual que déficit en el conocimiento de la transmisión de la enfermedad, en los principales factores de riesgo de contagio y en las medidas de prevención. Esto amerita una revisión del plan de estudio en la que se incluyan las patologías más prevalentes del país, específicamente la TB como realidad de salud pública nacional y mundial, y fomentando el autocuidado, ya que los estudiantes del área de la salud, al ser futuros profesionales estarán encargados de atender a pacientes, algunos de ellos con la enfermedad. De ahí la importancia del conocimiento actualizado sobre la TB y su tratamiento, el fomento de las medidas de bioseguridad e identificar a los pacientes sintomáticos respiratorios como posibles fuentes de contagio.

En este sentido, cuando el equipo de salud presenta insuficientes conocimientos de la TB, transmisión y tratamiento, disminuye la percepción del riesgo, lo que dificulta la eliminación de la TB a nivel general y las medidas de control planteadas por cada país, aumentando las tasas de enfermedad, incumplimiento del tratamiento, aparición de farmacorresistencias e incremento en las cifras de mortalidad por TB.

Es necesario incluir en el plan de estudio de pregrado de las facultades de Odontología y Terapia respiratoria, actividades de actualización sobre la enfermedad y que los egresados se conviertan en personal de apoyo en la lucha contra la TB en nuestro país. Además de detectar oportunamente a los sintomáticos respiratorios, prevenir posible contagio, ya que un diagnóstico oportuno y el tratamiento adecuado garantiza la cura y el control de la enfermedad.

\section{Financiación}

Esta investigación fue financiada con recursos de la Fundación Universitaria Autónoma de las Américas.

\section{Conflicto de intereses}

Ninguno de los autores declara conflicto de intereses. 


\section{Referencias}

1. Delgado J, Cadena L, Tarazona G, Lozano C, Rueda E. Nivel de conocimientos sobre tuberculosis en dos comunidades del Área Metropolitana de Bucaramanga, Santander. CES Salud Pública. 2015;(6):42-53.

2. World Health Organization. Tuberculosis Nota descriptiva. [Cited 2018 abril 10] Available from: http://www.who.int/mediacentre/factsheets/fs104/es.

3. OMS. Informe mundial sobre la tuberculosis 2017.[citado el 12 de enero 2018]. Disponible en:http://www.who.int/ campaigns/tb-day/2018/exe_summary_ es.pdf.

4. INS:Lineamientos para el manejo programático de la tuberculosis farmacoresistente. 2013 [Consultado abril 5 de 2018]. Disponible en: https://www.minsalud.gov.co/sites/rid/Lists/BibliotecaDigital/ RIDE/VS/PP/ET/lineamientos-tb-farmacorresistente.pdf.

5. Vallejo P, Rodríguez J, Searle A, Farga V. Ensayo Xpert MTB/RIF en el diagnóstico de tuberculosis. Rev. chil. enferm. respir. 2015; 31(2):127-131.

6. Plan Decenal de Salud Pública.2012-2021. [Consultado abril 6 de 2018]. Disponible en:http://www.minsalud. gov.co/salud/publica/Paginas/salud-publica.aspx

7. Galán E, et al. Bioseguridad durante el Internado de Medicina en Hospitales de Trujillo - La Libertad 2010: a propósito de la muerte de un estudiante de medicina. Acta méd. Peruana, Lima. 2010; 27(2).

8. Cano C, Quintero M, Ruiz M. La tuberculosis como enfermedad laboral en los trabajadores del área de la salud. Ces.2015. [Consultado abril de 2018].

172 Disponible en: http://bdigital.ces.edu.co: 8080/repositorio/bitstream/10946/4329/1/ Tuberculosis_Enfermedad_Laboral.pdf.
9. Cruz O, Flórez E, Muñoz A. Conocimientos sobre tuberculosis en trabajadores de la salud en una localidad de Bogotá D. C. Av. enferm. 2011;29(1):143-151.

10. Ministerio de Ciencia e Innovación Instituto Carlos III de Salud Escuela Nacional de Medicina del Trabajo. Prevención y Control de la Tuberculosis en Trabajadores del Ámbito Sanitario. Disponible en: http:// gesdoc.isciii.es/gesdoccontroller?action=download\&id=14/09/2012-46db82522e.

11. Wilches $\mathrm{E}$, Hernández $\mathrm{N}$, Hernández O, Pérez C. Conocimientos, actitudes, prácticas y educación sobre tuberculosis en estudiantes de una facultad de salud. Rev Salud pública. 2016:18(1):129-141.

12. Antolinez C, Bello M, Romero L, Muñoz A. Instrumentos y herramientas de evaluación sobre conocimientos de tuberculosis Enferm. Glob (Murcia) 2017; 16(48) Doi http://dx.doi.org/10.6018/eglobal. 16.4.262831.

13. Kanjee Z, et al. Tuberculosis infection control in rural South Africa: survey of knowledge, attitude and practice in hospital staff. J Hosp Infect. 2011;79(4):333338. doi:10.1016/j.jhin.2011.06.017.

14. World Health Organization: Policy on TB Infection Controls in Health-Care Facilities, Congregate Settings and Households [Internet] [Consultado 2017 dic 12] Available from: http://apps.who.int/iris/bitstr eam/10665/44148/1/9789241598323_ eng.pdf

15. Torres E, Barra M, Muñoz A. Conocimientos y actitudes de las medidas de bioseguridad en estudiantes de odontología en Puno. Evid Odontol Clin. Nov. 2015; 1:1. Disponible en:https://uancv. edu.pe/revistas/index.php/EOC/article/ view/113/93.

16. Hidalgo $\mathrm{P}$, Moreno $\mathrm{A}$, Roldán T. Tuberculosis, un riesgo presen- 
te para los trabajadores en el área de la salud. Universitas Médica[Internet].2011;52(2):227-236.

17. Ortega Barón GL, Rodríguez Quesada PA, Jiménez Beltrán EC, Muñoz Sánchez Al. Conocimientos sobre tuberculosis en estudiantes de enfermería de una universidad colombiana. Rev Univ Ind Santander. 2015; 47(3): 261-270.

18. Acosta C, Acosta G. Plan Estratégico Colombia Libre de Tuberculosis 20102015 para la expansión y fortalecimiento de la estrategia alto a la TB: vivamos sin tuberculosis, todos somos parte de la so- lución. Ministerio de la Protección Social. Diciembre de 2009.

19. Abreu Suárez CG, González Valdés JA, Muñoz Peña R, Solar Salaverri LA, Marchena Béquer JJ. La preparación del estudiante de Medicina para la eliminación de la tuberculosis. Educ Médica Súper. 2013; 27(1): 38-45.

20. Organización Panamericana de la Salud (OPS). Enseñanza de la tuberculosis en las facultades de salud: informe de una consulta de expertos. Cartagena, Colombia; 2004: 1-39. 